\title{
miR-145-5p Mimic Inhibits Bone Metastasis of Prostate Cancer Via the Regulation of Epithelial Mesenchymal Transition
}

Bingfeng Luo ( $\sim$ luobf@hku-szh.org )

University of Hong Kong-Shenzhen Hospital https://orcid.org/0000-0002-3604-4270

\section{Yuan Yuan}

University of Hong Kong-Shenzhen Hospital

Jian Hou

University of Hong Kong-Shenzhen Hospital

Guanming Kuang

University of Hong Kong-Shenzhen Hospital

\section{Ping Li}

University of Hong Kong-Shenzhen Hospital

Yifei Zhu

University of Hong Kong-Shenzhen Hospital

Xiang Yi

University of Hong Kong-Shenzhen Hospital

Yaping Xing

University of Hong Kong-Shenzhen Hospital

Richard Lo

University of Hong Kong-Shenzhen Hospital

Zhenquan Lu

University of Hong Kong-Shenzhen Hospital

\section{Research Article}

Keywords: prostate cancer bone metastasis, microRNAs, mimics, miR-145-5p, epithelial mesenchymal transition, apoptosis

Posted Date: July 8th, 2021

DOl: https://doi.org/10.21203/rs.3.rs-678540/v1

License: (c) (1) This work is licensed under a Creative Commons Attribution 4.0 International License. Read Full License 


\section{Abstract}

Background: The bone is the most common site of distant metastasis in prostate cancer. However, treatments for the bone metastasis of prostate cancer remain unsatisfactory. MicroRNAs (miRNAs) are small noncoding RNAs that play a variety of critical roles in tumor development and progression. Studies have confirmed that miRNA mimics could regulate the response to therapy in many cancers.

Methods: In this study, a set of forty-four miRNAs were reduced in prostate cancer patients with bone metastases by high-throughput sequencing analysis. Wound healing, transwell assays and western blotting analysis were used to explore the role of miRNA mimic in prostate cancer bone metastasis.

Results: Further gene ontology and pathway analysis showed that these miRNAs target genes are mainly involved in cellular metabolic process, intracellular membrane-bounded organelle, as well as proteoglycans in cancer and focal adhesion. Therefore, these down-regulated miRNAs may play a key role for prostate cancer bone metastasis treatment, including hsa-miR-221-3p, hsa-miR-222-3p, hsa-miR133a-3p, hsa-miR-222-5p, hsa-miR-204-3p, hsa-miR-145-5p, hsa-miR-3681-5p, hsa-miR-184, hsa-miR-144$3 p$, hsa-miR-204-5p, and hsa-miR-221-5p. To further investigate the role of these miRNA mimics on prostate cancer bone metastasis, miR-145-5p was randomly selected for validation. Bioinformatics analysis showed that miR-145-5p target genes significantly affected TGF-beta and adherens junction signaling pathway. Wound healing and transwell assays and western blotting analysis revealed that miR145-5p mimic inhibited proliferation, migration and invasion. Importantly, miR-145-5p mimic increased the expression of E-cadherin and reduced the expression of matrix metalloproteinase 2 and 9 . These results revealed that miR-145-5p mimic mediated epithelial mesenchymal transition. Meanwhile, miR-145$5 p$ mimic enhanced the level of caspase 9 , which is an important promoter of apoptosis.

Conclusions: These results indicate that miR-145-5p mimic could inhibit the progress of prostate cancer bone metastasis via regulation of epithelial mesenchymal transition. In addition, miR-145-5p mimic could induce the apoptosis of prostate cancer cells with bone metastases. In summary, the miR-145-5p mimic is expected to become a novel strategy for the treatment of tumor metastasis.

\section{Introduction}

Seventy percent of patients with metastatic prostate cancer have bone metastasis [1]. The bone is the most common site of distant metastasis in prostate cancer. Unfortunately, once primary tumor spreads to bone, it is rarely cured. The mechanism of bone metastasis development is extremely complex. In 1889, Stephen Page first described the complexity of bone and proposed that tumor cells have a tendency migrating to some organs, where they are "sown" into favorable "soil" and eventually develop into metastatic lesions. It is now clear that the "soils" of different metastatic sites are unique [2]. For example, metastases are common in vertebrae, ribs, pelvis bones and the ends of long bones. However, it's rarely observed in the bones of the hands or feet [3]. Therefore, it is of great significance for the treatment of bone metastasis of prostate cancer to identify and verify the genes that mediate bone metastasis. 
Studies have shown that osteonectin, transforming growth factor- $\beta$ (TGF $\beta$ ), C-X-C motif chemokine ligand 12 (CXCL12) and vascular endothelial growth factor (VEGF) are involved in the extravasation and guidance of prostate cancer cells to bone; in addition, cadherin 11 plays an important role in the interaction between prostate cancer cells and osteoblasts [4]. The N-terminal fragment of urokinase can be used as an effective stimulator of prostate cancer cell proliferation [5]. FGF, IGF and TGF $\beta$ from the tumor microenvironment play regulatory roles in the survival and proliferation of prostate cancer cells [6]. Prostate transmembrane protein androgen inducible 1 (PMEPA1) has been identified as another regulator of transforming growth factor- $\beta$ (TGF- $\beta$ ) signal transduction and inhibits bone metastasis of prostate cancer. Decreased PMEPA1 expression was found in patients with metastatic prostate cancer, and low PMEPA1 expression was associated with decreased metastasis-free survival [7]. Studies have found that prostate-specific antigen (PSA) is a kallikrein-type serine protease that can cleave and activate parathyroid hormone-related peptide (PTHrP), thus accelerating the growth of bone metastases [8]. An important finding is that CXCR4 blocking peptides inhibited the bone metastasis of prostate cancer cells, and this signaling axis is involved in adhesion of prostate cancer cells to bone marrow endothelial cells [9]. An analysis of prostate adenocarcinoma samples in the cancer genome atlas (TCGA) (360 tumors, 51 normal tissues) showed that in prostate carcinoma, the RNA levels of BSP CX chemokine receptor 4 (CXCR4), prostate transmembrane protein androgen induced 1 (PMEPA1) and bone sialoprotein (BSP) were differentially expressed in tumor tissues in comparison to their corresponding matching normal tissues [10]. However, the clinical application of these genes in the treatment of bone metastasis of prostate cancer requires further study.

MicroRNAs (miRNAs), small noncoding RNAs, play a variety of critical roles in tumor development, progression and response to therapy by regulating gene expression [11]. miRNAs can be used as biomarkers and as a novel treatment in many cancers [12]. miRNAs also play a key role in epithelial mesenchymal transition, osteomimicry and osteoblast and osteoclast function $[13,14]$. miRNA dysregulation is the cause of many cancers. miRNAs play roles as tumor suppressors or oncogenes. miRNA mimics and miRNA targeting molecules (antimirs) have shown promise in preclinical development. Some miRNA-targeted therapies have entered the clinical development stage. Tumor suppressor miRNA miR-34 mimics have reached the first stage of clinical trials in the treatment of cancer [15]. Molecules targeting miRNAs (antimiRs), including those that target miR-122, have reached the second stage of clinical trials in the treatment of hepatitis [16]. miR-203 was found to act as a suppressor of bone metastasis. In the mouse model of bone metastasis, ectopic expression of miR-203 in the bone metastatic prostate cell line PC3 can induce morphological changes from a fibroblast-like phenotype to an epithelial-like phenotype and reduce the metastatic tendency [17]. Therefore, it is of potential significance to search for downregulated miRNAs in prostate cancer bone metastases, and these miRNAs could act as potential biomarkers related to the occurrence of bone metastases of prostate cancer. miRNA mimics may serve as novel treatments of prostate cancer bone metastases.

\section{Methods}




\section{Patients}

Patients diagnosed with prostate cancer were enrolled at the University of Hong Kong-Shenzhen Hospital. These patients (mean age of 65.3 years, age range of 59-70 years) underwent pelvic magnetic resonance imaging (MRI) and bone scan before prostate tumor biopsy. Immunohistochemical staining confirmed the diagnosis of prostate cancer. These patients were divided into two groups, namely, prostate cancer with bone metastases and prostate cancer without bone metastases, according to bone scan diagnosis. Informed consents were obtained from patients regarding the use of the prostate cancer tissue samples for this study. The project was approved by the University of Hong Kong-Shenzhen Hospital Research Ethics Committee (No. [2019]260). All the work was conducted in accordance with the Declaration of Helsinki (1964).

\section{Total Rna Extraction And Sequencing}

Total RNA extraction and sequencing

Total RNA was isolated from prostate cancer tissues using TRIpure Total RNA Extraction Reagent (ELK Biotechnology, Wuhan, China) according to the manufacturer's protocol. RNA pellets were washed in $75 \%$ ethanol, air dried, resuspended in RNase-free water supplemented with RNase inhibitor (Thermo Fisher Scientific, Vienna, Austria) and stored at $-80^{\circ} \mathrm{C}$. The RNA yield and purity were measured using an Agilent

2100 Bioanalyzer (Agilent Technologies Inc., California, America). Sequencing libraries were prepared at the Chi-biotech Corporation (Shenzhen, China) followed by sequencing with Illumina HiSeq2500. The quality of the microRNA sequencing reads was assessed with FastQC, and the reads were aligned with the human genome reference (GRCh38, Ensembl release 76) with bowtie. The expression of miRNAs was quantified using HTSeq version 0.6.1p1 according to miRbase (http://www.mirbase.org/) for human miRNAs.

\section{Differential Expression Analyses Of Mirnas}

DESeq2 (version 1.20.0) was used to analyze the differential expression of miRNAs. The BenjaminiHochberg method was used to calculate false discovery rate (FDR)-adjusted p-values, accounting for multiple testing correction. miRNAs were considered differentially expressed when the FDR adjusted pvalues were less than 0.01 and the fold-change was more than the threshold of 2 . The miRTarBase database was used to predict miRNA target genes. The enrichment analysis used KOBAS annotation, which includes Kyoto Encyclopedia of Genes and Genomes (KEGG) pathway analyses and Gene Ontology (GO). Cytoscape software V3.2.1 was used to generate the miRNA-mRNA interaction network, and the data output was received in Excel spreadsheets.

\section{Quantitative Reverse Transcription Pcr Validation Of Mirna}


To validate the sequencing results, we assessed the miRNAs with differential expression levels in 20 prostate cancer samples (patients with bone metastases, $n=10$, and patients without bone metastases, $n=10)$ using quantitative reverse transcription PCR (RT-qPCR). cDNA was synthesized from total RNA using the EntiLink ${ }^{\mathrm{Tm}} 1$ st Strand cDNA Synthesis Kit (ELK Biotechnology, Wuhan, China). PCR was performed using EnTurbo ${ }^{\text {TM }}$ SYBR Green PCR SuperMix (ELK Biotechnology). All reactions were incubated in a 96 -well plate at $95^{\circ} \mathrm{C}$ for $3 \mathrm{~min}$, followed by 40 cycles of $95^{\circ} \mathrm{C}$ for $10 \mathrm{~s}, 58^{\circ} \mathrm{C}$ for $30 \mathrm{~s}$, and $72^{\circ} \mathrm{C}$ for 30 s. U6 was used as the internal control. The stem-loop primers were as follows: U6 (NR_004394.1), forward primer, CTCGCTTCGGCAGCACAT, and reverse primer, AACGCTTCACGAATTTGCGT; hsa-miR-145-5p (MIMAT0000437), forward primer, CAGTTTTCCCAGGAATCCCT, and reverse primer, CTCAACTGGTGTCGTGGAGTC. The quantification of miRNA expression was performed by the double delta $\mathrm{Ct}$ method.

\section{Cell Culture And Mirna Mimic Transfection}

The prostate cancer bone metastatic cell line PC-3 was maintained in Dulbecco's modified Eagle's medium (Gibco, California, America), including 10\% FBS (Gibco), $100 \mathrm{U} / \mathrm{mL}$ penicillin, and $100 \mu \mathrm{g} / \mathrm{mL}$ streptomycin in a humidified $\mathrm{CO}_{2}$ incubator. Cells $\left(3 \times 10^{5}\right)$ were plated in 6-well plates and transfected with a hsa-miR-145-5p mimic (forward: GUCCAGUUUUCCCAGGAAUCCCU, and reverse: CUGGUCUUUUGGGUCCUUUGGGU) and control mimic (forward: UUUGUACUACACAAAAGUACUG and reverse: AAACAUGAUGUGUUUUCAUGAC) using Lipofectamine 2000 (Invitrogen, California, America) according to the manufacturer's protocol.

\section{Proliferation, Migration And Invasion Assay}

The cell migration activity after miRNA mimic treatment was investigated using an in vitro proliferation, migration and invasion assay. Cells were seeded in a 96-well plate for proliferation assay. CCK8 (Cell Counting Kit-8, Biosharp) reagent was added to cell culture and absorbance was measured at $450 \mathrm{~nm}$ after $1 \mathrm{~h}$ of incubation. For wound healing migration assay, $3 \times 10^{5}$ cells/well were grown in a 24-well microplate for 16-18 h. The cells were then serum-starved overnight. The centers of the cell monolayers were then scraped in a straight line by pipette tip to create a wound, and then, the cells were washed with PBS. Medium was added, and the cells were incubated for $24 \mathrm{~h}$. The wound closure was photographed by an inverted microscope with a digital microscope camera. The symmetry of the median distance between both borderlines was used to calculate the migration index. Invasion assays were carried out in transwell chambers separated by a semipermeable membrane with an $8-\mu \mathrm{m}$ pore size (Corning). After transfection as described above, the cells were detached from the culture plates and resuspended in serum-free medium containing $0.1 \% \mathrm{BSA}$. The cells were allowed to migrate to the lower chamber for $24 \mathrm{~h}$ at $37^{\circ} \mathrm{C}$ in $5 \% \mathrm{CO}_{2}$. The cells were removed from the chamber, the medium was removed by washing with PBS, and the cells were fixed with paraformaldehyde for 20 min, washed with PBS again, and stained with $0.1 \%$ 
crystal violet for 10 min; PBS was used to wash the extra crystal violet from the cells, and the side on which the cells were not seeded was photographed under the inverted microscope.

\section{Western Blotting Analysis}

Protein was extracted from cells with radioimmunoprecipitation assay (RIPA) buffer and analyzed by western blotting analysis. The proteins were resolved by SDS/PAGE with 10\% Bis-Tris (Invitrogen, America) and transferred to nitrocellulose membranes. The membrane was incubated in a $5 \%$ solution of nonfat milk for $1 \mathrm{~h}$ at $4^{\circ} \mathrm{C}$. After overnight incubation with the primary antibody (Abcam, America) at $4^{\circ} \mathrm{C}$ (Table 1), the blots were washed in Tween-TBS for $20 \mathrm{~min}$ and incubated with the secondary antibody. The blots were washed in T-TBS for 20 min and detected with an electroluminescence kit (ASPEN, Wuhan, China). GAPDH staining was used as the loading control.

Table 1

The information of the primary antibodies

\begin{tabular}{|lll|}
\hline Name & Information & ID \\
\hline MMP9 & Abcam & ab137867 \\
\hline MMP2 & Abcam & ab97779 \\
\hline E-cadherin & Abcam & ab1416 \\
\hline caspase 9 & Abcam & ab115792 \\
\hline GAPDH & Abcam & ab8245 \\
\hline
\end{tabular}

\section{Statistical analysis}

All experiments were performed in triplicate incubations. Data were analyzed as mean \pm SD of three independent experiments. Data were analyzed by two-tailed unpaired Student's t-test between any two groups. A value of $P<0.05$ was considered statistically significant. Statistical tests were performed using GraphPad software v. 4.1 (CA, USA).

\section{Results}

\section{miRNA analysis in patients diagnosed with prostate cancer}

All patients were finally diagnosed by pelvic magnetic resonance imaging (MRI), whole-body-bone scan imaging, hematoxylin-eosin (HE) and periodic acid-schiff (PAS) staining of pathological sections (Fig. 1). Firstly, high-throughput sequencing analysis of microRNAs was performed with five prostate cancer tissues and three prostate cancer bone metastases tissues. A set of 122 microRNAs were previously identified as modulators of bone metastasis in prostate cancer. The expression of these miRNAs $(n=78)$ was upregulated while the expression of 44 miRNAs was decreased in prostate cancer with bone 
metastases compared to prostate cancer without metastasis (Fig. 2A). In addition, to explore the roles of these differentially expressed miRNAs in prostate cancer with bone metastasis, Kyoto Encyclopedia of Genes and Genomes (KEGG) pathway analyses were conducted to explore the function of the predicted mRNA targets (binding site predicted targets from miRTarBase) of these related miRNAs (Fig. 2B). The results showed that these miRNAs regulate many tumor-related signaling pathways, such as the MAPK signaling pathway and focal adhesion, the most significant of which are proteoglycans in the cancer signaling pathway. According to the Gene Ontology (GO) analysis system, three major functional categories (biological process, cellular component, and molecular function) were used to classify these predicted mRNA targets (Fig. 2C-E). Genes involved in "nucleobase-containing compound biosynthetic" and "cellular metabolic process" groups were notably found in the biological process category. Among the cellular components, "intracellular membrane-bound organelle" and "cytoplasm" were the most common categories. In the category of molecular function, a significant proportion of clusters were assigned to "heterocyclic compound binding" and "transcription factor binding". These findings indicate that these miRNAs whose expression is suppressed potentially play an important role in prostate cancer with bone metastasis.

\section{miR-145-5p is expressed at low levels in prostate cancer tissues with bone metastasis and the bone metastatic prostate cancer cell line PC-3.}

To verify the results of high-throughput sequencing, hsa-miR-145-5p was randomly selected (Fig. 3A). The expression level of miR-145-5p in ten prostate cancer patients with bone metastasis and ten prostate cancer patients without metastasis was detected by RT-qPCR respectively. Compared with those prostate cancer tissues without metastasis, the expression of miR-145-5p in prostate cancer tissues with bone metastasis was significantly decreased (Fig. 3B). miR-145-5p was also reduced in bone metastatic prostate cancer cell line (PC3) compared with that in prostate cancer without metastatic cells (22RV1) and normal prostate epithelial cells (RWPE-1) (Fig. 3C). Next, to further determine the possible biological impact of miR-145-5p on the pathogenesis of prostate cancer bone metastasis, the miR-145-5p miRNA target genes from miRTarBase were used to predict the possible enrichment pathways and related biological processes using KEGG and GO analysis. In total, 30 KEGG pathways were significantly enriched, with the numbers of miR-145-5p targets ranging from 3 to 7 molecules per enriched pathway (Fig. 3D). Among the enriched biological pathways were MAPK signaling (hsa04010), TGF-beta signaling (hsa04350), adherens junction signaling (hsa04520), proteoglycans in cancer signaling (hsa05205), cellular senescence (hsa04218), VEGF signaling (hsa04370) and FoxO signaling (hsa04068), which were previously described as cancer pathogenesis-associated pathways. To obtain deeper insights into the putative function of miR-145-5p, network diagrams involving the predicted target genes were generated, and these diagrams showed a greater relationship with miR-145-5p, paxillin (PXN), NEDD9, SMAD3, SMAD5 and TGFBR2 (Fig. 3E). GO analysis also revealed that tumor metastasis-related processes, including cell adhesion, cell migration, cell motility and cellular response to endogenous stimulus, occupied a very prominent place in biological processes (Fig. 3F). Furthermore, the cytoplasm, intracellular system and nucleoplasm were all regulated in the cellular component category (Fig. 3G). Especially in the molecular function term, protein-containing complex binding, enzyme binding, kinase 
binding and transcription regulatory region DNA binding all play important roles in cell proliferation (Fig. 3H). The results suggested that miR-145-5p regulated these target genes, possibly exerting effects of great significance on the process of prostate cancer bone metastasis.

\section{miR-145-5p inhibits proliferation, migration and invasion via epithelial mesenchymal transition.}

To further investigate the biological role of miR-145-5p in bone metastasis of prostate cancer, exogenous miR-145-5p mimic and normal control miRNA mimic were transfected into bone metastatic prostate cancer PC-3 cells. The RT-qPCR results showed that the expression of miR-145-5p was increased in in PC3 cells treated with the miR-145-5p mimic compared with that in PC-3 cells treated with the control mimic (Fig. 4A). Cell counting kit-8, scratch and transwell assays were used to assess the proliferation, migration and invasion of PC-3 cells with miR-145-5p mimic (Fig. 4B-F). The results showed that the migration and invasion of PC-3 cells were inhibited in the overexpressed miR-145-5p group compared to the control group. To explore the effect of miR-145-5p on epithelial mesenchymal transition in PC-3 cells, the levels of MMP-2, MMP-9 and E-cadherin were assessed via western blotting analysis (Fig. 4G-H). The results revealed that upregulating miR-145-5p expression increased the expression of the epithelial marker E-cadherin and reduced the expression of matrix metalloproteinase 2 and 9 . Moreover, caspase 9 was activated by miR-145-5p, indicating that miR-145-5p promoted apoptosis in PC-3 cells. These results indicate that the miR-145-5p mimic may be an effective molecule for the treatment of bone metastasis of prostate cancer and would inhibit the occurrence of bone metastasis of prostate cancer.

\section{Discussion}

At present, the treatments for the bone metastasis of prostate cancer include hormonal therapy, chemotherapy, and radiotherapy. These approaches can reduce pain and complications (such as fractures), and improve quality of life [18-20]. However, the prognosis of prostate cancer patients with bone metastasis is poor. More strategies are needed to strengthen the diagnosis and treatment of bone metastasis of prostate cancer. miRNAs are versatile molecules that have been used to increase the specificity and therapeutic efficacy of various treatments for cancers. In this study, we screened deregulated miRNAs in prostate cancer bone metastasis and explored the possibility of developing the miR-145-5p mimic into a novel therapy to treat prostate cancer bone metastases.

In this study, we found that the top 11 significantly changed miRNAs (hsa-miR-221-3p, hsa-miR-222-3p, hsa-miR-133a-3p, hsa-miR-222-5p, hsa-miR-204-3p, hsa-miR-145-5p, hsa-miR-3681-5p, hsa-miR-184, hsamiR-144-3p, hsa-miR-204-5p, and hsa-miR-221-5p) were deregulated in prostate cancer with bone metastasis malignant transformation. It has been reported that miR-221 may be a potential prognostic biomarker of hepatocellular carcinoma, lung cancer, and breast cancer and a critical regulator of therapeutic susceptibility in breast cancer [21-23]. The active cells in bone include osteoblasts, osteoclasts, hematopoietic cells, adipocytes and various immune cells. Soudeh et al reported that miR221 participates in the differentiation of hematopoietic stem cells [24]. In addition, the low expression of miR-221 and miR-222 increases kit protein synthesis, which leads to the development of early 
erythroblastic cells [25]. These findings showed that miR-221 and miR-222 may have a certain effect on the bone hematopoietic stem cells in prostate cancer bone metastasis. OIP5-AS1, a modulator of epithelial-mesenchymal transition, acts as a competing endogenous RNA for miR-204-5p and increases in nearly all kinds of neoplastic tissues [26]. These results suggest that it is possible to cure many cancers by elevating the expression of miR-204-5p, causing poor expression of OIP5-AS1. Hsa-miR-3681-5p has been found to act as a superenhancer and as a repressor of VNTR activity in the 3' UTR of SHISA7 [27]. This finding may be related to the occurrence of prostate bone metastasis with low expression of miR3681-5p. miR-133a-3p has a positive impact on the formation of osteoclasts and/or their bone resorption capacity through p38 MAP-kinase [28]. miR-184 has been found to inhibit tumor invasion, migration and metastasis in nasopharyngeal carcinoma by targeting notch2 [29]. miR-144-3p was deregulated in osteosarcoma tissues, and miR-144-3p overexpression inhibited the migration and EMT of osteosarcoma cells [30]. These miRNAs may also play an important role in bone metastasis of prostate cancer, and their mimics may be able to cure bone metastasis of prostate cancer.

Ozen $\mathrm{M}$ et al found that miR-145-5p may be an important regulator of SOX2 and inhibited the proliferation of prostate cancer cells. In our study, miR-145-5p was expressed at a low level in prostate cancer bone metastatic tissues and cell lines. The miR-145-5p mimic also inhibited the migration and invasion of prostate cancer bone metastatic cells. In addition, miR-145-5p may suppress the invasion of glioblastoma cells and the progression of osteosarcoma [31,32]. Epithelial to mesenchymal transition is characterized by the loss of the typical epithelial phenotype and the acquisition of mesenchymal characteristics, and overactivation of epithelial mesenchymal transition promotes cancer metastasis [33]. Matrix metalloproteinases (MMPs), including MMP-2 and MMP-9, have been proven to favor cancer cell invasion and metastasis during epithelial mesenchymal transition [34]. E-cadherin is a well-known tumor suppressor protein, and the loss of its expression occurs frequently during tumor metastasis [35]. Caspase 9 is an important promoter of apoptosis [36]. In our study, miR-145-5p reduced the expression of MMP-2 and MMP-9 and increased the expression of E-cadherin and caspase 9 in PC-3 cells. This suggests that miR-145-5p inhibits PC-3 cell metastasis and promotes PC-3 cell apoptosis. Moreover, the expression of hsa-miR-145-5p is also decreased in tumor transformational apocrine and spindle cells compared to in situ carcinoma or nonttumor structures [37]. This indicates that miR-145-5p has a potential regulatory effect on cancer metastasis. Consequently, the miR-145-5p mimic is expected to become a new approach for the treatment of tumor metastasis.

\section{Conclusion}

In this study, we identified differentially expressed miRNA profiles between prostate cancer with bone metastasis and prostate cancer without bone metastasis. The mimics of these decreased miRNAs in prostate cancer with bone metastasis, including hsa-miR-221-3p, hsa-miR-222-3p, hsa-miR-133a-3p, hsamiR-222-5p, hsa-miR-204-3p, hsa-miR-145-5p, hsa-miR-3681-5p, hsa-miR-184, hsa-miR-144-3p, hsa-miR204-5p, and hsa-miR-221-5p, may play an important role in the treatment of prostate cancer bone metastasis. Importantly, the results revealed that the miR-145-5p mimic could become a novel strategy for the treatment of prostate cancer bone metastasis by regulating epithelial mesenchymal transition and 
inducing prostate cancer bone metastatic cells apoptosis (Fig. 5). In a word, miR-145-5p mimic need to be paid close attention to the treatment of prostate cancer patients with bone metastasis.

\section{Declarations}

\section{Acknowledgements}

\section{Ethics approval and consent to participate}

The following information was supplied relating to ethical approvals: The University of Hong KongShenzhen Hospital Research Ethics Committee approved this research (No. [2019]260).

\section{Consent for publication}

Informed consents were obtained from patients regarding the use of the prostate cancer tissue samples and any individual person's data for this study.

\section{Availability of data and materials}

The following information was supplied regarding data availability at the Zenodo, DOI:10.5281/zenodo.4972098.

\section{Competing Interests}

All authors declare that there is no conflict of interest in this work.

\section{Funding}

This research is supported by the project "High Level Hospital Program, Health Commission of Guangdong Province, China (No. HKUSZH201901024). The funders had no role in study design, data collection and analysis, decision to publish, or preparation of the manuscript.

\section{Author contributions}

Zhenquan Lu and Bingfeng Luo conceived and designed the project; Bingfeng Luo wrote the paper; Yuan Yuan, Xiang Yi, Jian Hou, Yaping Xing and Ping Li acquired the data; Guanming Kuang and Yifei Zhu analysed and interpreted the data; Richard Lo modified the language of the article.

\section{References}

1. Fornetti J, Welm AL, Stewart SA. Understanding the Bone in Cancer Metastasis. Journal of bone mineral research: the official journal of the American Society for Bone Mineral Research. 2018;33(12):2099-113. 
2. Fidler IJ. The pathogenesis of cancer metastasis: the 'seed and soil' hypothesis revisited. Nature reviews Cancer. 2003;3(6):453-8.

3. DiNatale A, Fatatis A. The Bone Microenvironment in Prostate Cancer Metastasis. Adv Exp Med Biol. 2019;1210:171-84.

4. Chu K, Cheng CJ, Ye X, Lee YC, Zurita AJ, Chen DT, Yu-Lee LY, Zhang S, Yeh ET, Hu MC, et al. Cadherin-11 promotes the metastasis of prostate cancer cells to bone. Molecular cancer research: MCR. 2008;6(8):1259-67.

5. Sheng S. The urokinase-type plasminogen activator system in prostate cancer metastasis. Cancer Metastasis Rev. 2001;20(3-4):287-96.

6. Buenrostro D, Park SI, Sterling JA. Dissecting the role of bone marrow stromal cells on bone metastases. BioMed research international. 2014;2014:875305.

7. Fournier PG, Juarez P, Jiang G, Clines GA, Niewolna M, Kim HS, Walton HW, Peng XH, Liu Y, Mohammad KS, et al. The TGF-beta Signaling Regulator PMEPA1 Suppresses Prostate Cancer Metastases to Bone. Cancer cell. 2015;27(6):809-21.

8. Kaplan RN, Psaila B, Lyden D. Bone marrow cells in the 'pre-metastatic niche': within bone and beyond. Cancer Metastasis Rev. 2006;25(4):521-9.

9. Houshmand P, Zlotnik A. Targeting tumor cells. Curr Opin Cell Biol. 2003;15(5):640-4.

10. Weidle UH, Birzele F, Kollmorgen G, Ruger R. Molecular Mechanisms of Bone Metastasis. Cancer genomics proteomics. 2016;13(1):1-12.

11. Iorio MV, Croce $\mathrm{CM}$. MicroRNA dysregulation in cancer: diagnostics, monitoring and therapeutics. A comprehensive review. EMBO molecular medicine. 2012;4(3):143-59.

12. Acunzo M, Romano G, Wernicke D, Croce CM. MicroRNA and cancer-a brief overview. Advances in biological regulation. 2015;57:1-9.

13. Brabletz T. To differentiate or not-routes towards metastasis. Nature reviews Cancer. 2012;12(6):425-36.

14. Croset M, Santini D, luliani M, Fioramonti M, Zoccoli A, Vincenzi B, Tonini G, Pantano F. MicroRNAs and bone metastasis: a new challenge. Molecules. 2014;19(7):10115-28.

15. Bouchie A. First microRNA mimic enters clinic. Nature biotechnology. 2013;31(7):577.

16. Rupaimoole R, Slack FJ. MicroRNA therapeutics: towards a new era for the management of cancer and other diseases. Nature reviews Drug discovery. 2017;16(3):203-22.

17. Saini S, Majid S, Yamamura S, Tabatabai L, Suh So, Shahryari V, Chen Y, Deng G, Tanaka Y, Dahiya R. Regulatory Role of mir-203 in Prostate Cancer Progression and Metastasis. Clinical cancer research: an official journal of the American Association for Cancer Research. 2011;17(16):5287-98.

18. Saad F, Gleason DM, Murray R, Tchekmedyian S, Venner P, Lacombe L, Chin JL, Vinholes JJ, Goas $\mathrm{JA}$, Chen $\mathrm{B}$, et al. A randomized, placebo-controlled trial of zoledronic acid in patients with hormonerefractory metastatic prostate carcinoma. J Natl Cancer Inst. 2002;94(19):1458-68. 
19. Sartor O, Reid RH, Hoskin PJ, Quick DP, Ell PJ, Coleman RE, Kotler JA, Freeman LM, Olivier P. Quadramet 424Sm10/11 Study G: Samarium-153-Lexidronam complex for treatment of painful bone metastases in hormone-refractory prostate cancer. Urology. 2004;63(5):940-5.

20. Tu SM, Millikan RE, Mengistu B, Delpassand ES, Amato RJ, Pagliaro LC, Daliani D, Papandreou CN, Smith TL, Kim J, et al. Bone-targeted therapy for advanced androgen-independent carcinoma of the prostate: a randomised phase II trial. Lancet. 2001;357(9253):336-41.

21. Dobre EG, Dinescu S, Costache M. Connecting the Missing Dots: ncRNAs as Critical Regulators of Therapeutic Susceptibility in Breast Cancer. Cancers 2020, 12(9).

22. Smolarz M, Widlak P. Serum Exosomes and Their miRNA Load-A Potential Biomarker of Lung Cancer. Cancers 2021, 13(6).

23. Xu J, An P, Winkler CA, Yu Y. Dysregulated microRNAs in Hepatitis B Virus-Related Hepatocellular Carcinoma: Potential as Biomarkers and Therapeutic Targets. Frontiers in oncology. 2020;10:1271.

24. Ghafouri-Fard S, Niazi V, Taheri M. Role of miRNAs and IncRNAs in hematopoietic stem cell differentiation. Non-coding RNA research. 2021;6(1):8-14.

25. Felli N, Fontana L, Pelosi E, Botta R, Bonci D, Facchiano F, Liuzzi F, Lulli V, Morsilli O, Santoro S, et al: MicroRNAs 221 and 222 inhibit normal erythropoiesis and erythroleukemic cell growth via kit receptor down-modulation. Proceedings of the National Academy of Sciences of the United States of America 2005, 102(50):18081-18086.

26. Ghafouri-Fard S, Dashti S, Farsi M, Hussen BM, Taheri M. A review on the role of oncogenic IncRNA OIP5-AS1 in human malignancies. Biomedicine pharmacotherapy = Biomedecine pharmacotherapie. 2021;137:111366.

27. Lee HE, Park SJ, Huh JW, Imai H, Kim HS: Enhancer Function of MicroRNA-3681 Derived from Long Terminal Repeats Represses the Activity of Variable Number Tandem Repeats in the 3' UTR of SHISA7. Molecules and cells 2020, 43(7):607-618.

28. Stephens E, Roy M, Bisson M, Nguyen HD, Scott MS, Boire G, Bouchard L, Roux S. Osteoclast signaling-targeting miR-146a-3p and miR-155-5p are downregulated in Paget's disease of bone. Biochimica et biophysica acta Molecular basis of disease. 2020;1866(10):165852.

29. Zhu HM, Jiang XS, Li HZ, Qian LX, Du MY, Lu ZW, Wu J, Tian XK, Fei Q, He X, et al: miR-184 Inhibits Tumor Invasion, Migration and Metastasis in Nasopharyngeal Carcinoma by Targeting Notch2. Cellular physiology and biochemistry: international journal of experimental cellular physiology, biochemistry, and pharmacology 2018, 49(4):1564-1576.

30. Cao J, Han X, Qi X, Jin X, Li X. TUG1 promotes osteosarcoma tumorigenesis by upregulating EZH2 expression via miR-144-3p. Int J Oncol. 2017;51(4):1115-23.

31. Kurogi R, Nakamizo A, Suzuki So, Mizoguchi M, Yoshimoto K, Amano T, Amemiya T, Takagishi S, lihara K. Inhibition of glioblastoma cell invasion by hsa-miR-145-5p and hsa-miR-31-5p cooverexpression in human mesenchymal stem cells. Journal of neurosurgery. 2018;130(1):44-55.

32. Li H, Pan R, Lu Q, Ren C, Sun J, Wu H, Wen J, Chen H. MicroRNA1455p inhibits osteosarcoma cell proliferation by targeting E2F transcription factor 3. Int J Mol Med. 2020;45(5):1317-26. 
33. Pastushenko I, Blanpain C. EMT Transition States during Tumor Progression and Metastasis. Trends in cell biology. 2019;29(3):212-26.

34. Scheau C, Badarau IA, Costache R, Caruntu C, Mihai GL, Didilescu AC, Constantin C, Neagu M. The Role of Matrix Metalloproteinases in the Epithelial-Mesenchymal Transition of Hepatocellular Carcinoma. Analytical cellular pathology. 2019;2019:9423907.

35. Na TY, Schecterson L, Mendonsa AM, Gumbiner BM. The functional activity of E-cadherin controls tumor cell metastasis at multiple steps. Proc Natl Acad Sci USA. 2020;117(11):5931-7.

36. Johnson CR, Jarvis WD. Caspase-9 regulation: an update. Apoptosis: an international journal on programmed cell death. 2004;9(4):423-7.

37. Koleckova M, Ehrmann J, Bouchal J, Janikova M, Brisudova A, Srovnal J, Staffova K, Svoboda M, Slaby O, Radova L, et al. Epithelial to mesenchymal transition and microRNA expression are associated with spindle and apocrine cell morphology in triple-negative breast cancer. Scientific reports. 2021;11(1):5145.

\section{Figures}




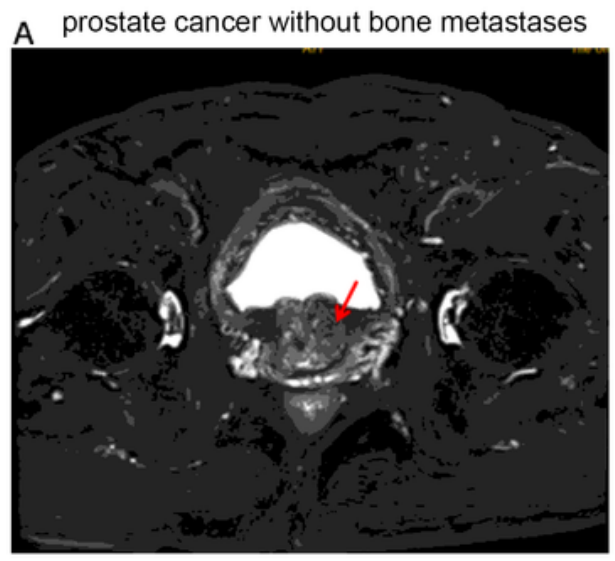

C prostate cancer without bone metastases
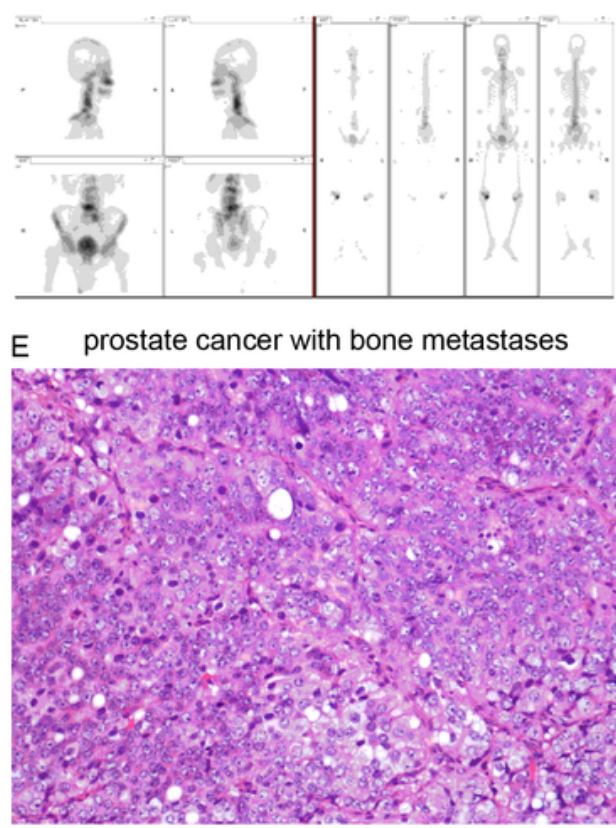

F prostate cancer with bone metastases

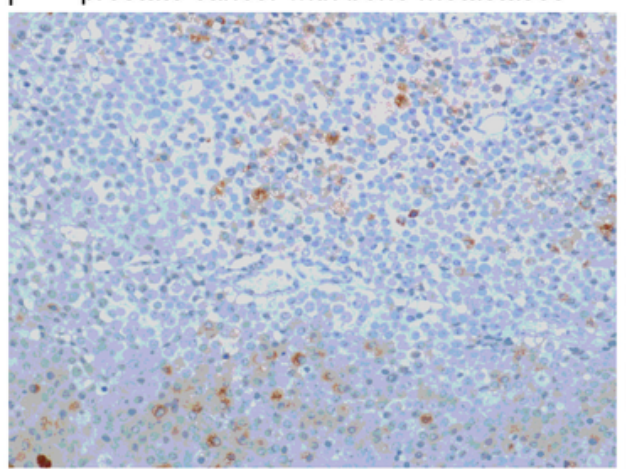

B prostate cancer with bone metastases

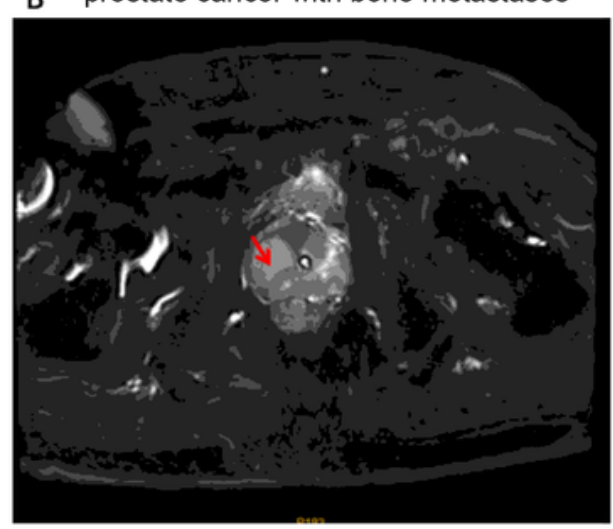

D prostate cancer with bone metastases

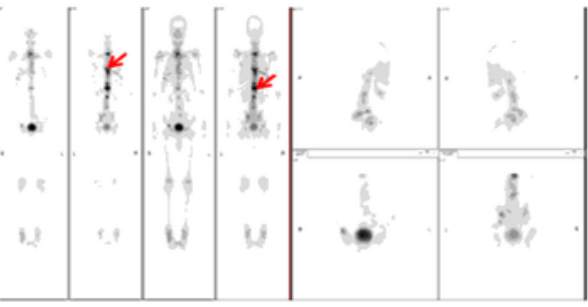

prostate cancer without bone metastases

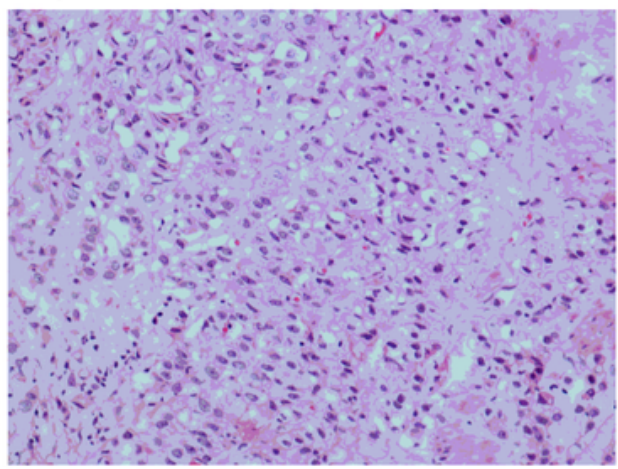

prostate cancer without bone metastases

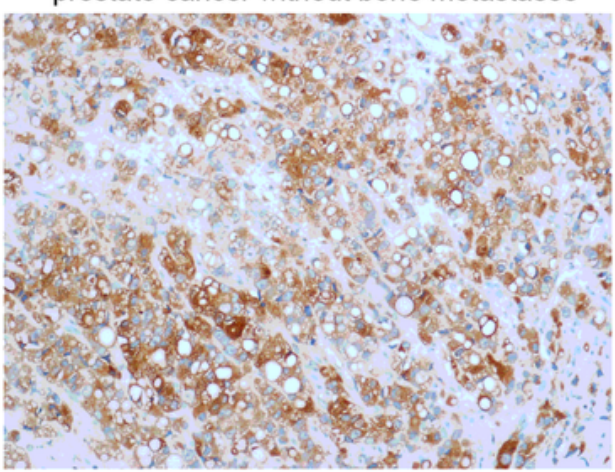

\section{Figure 1}

Diagnosis of prostate cancer with bone metastasis. (A) Pelvic magnetic resonance imaging (MRI) indicated a lesion of prostate cancer without bone metastases in the left lobe of the prostate (red arrow), as well as a lesion of prostate cancer with bone metastases in the right lobe of the prostate (red arrows). (B) Whole-body bone scan in the prostate cancer patients without bone metastases and prostate cancer 
patients with bone metastases (red arrows). (E-F) HE and PSA staining tissues morphology of prostate cancer with bone metastases and without bone metastases at objective 20X.

A

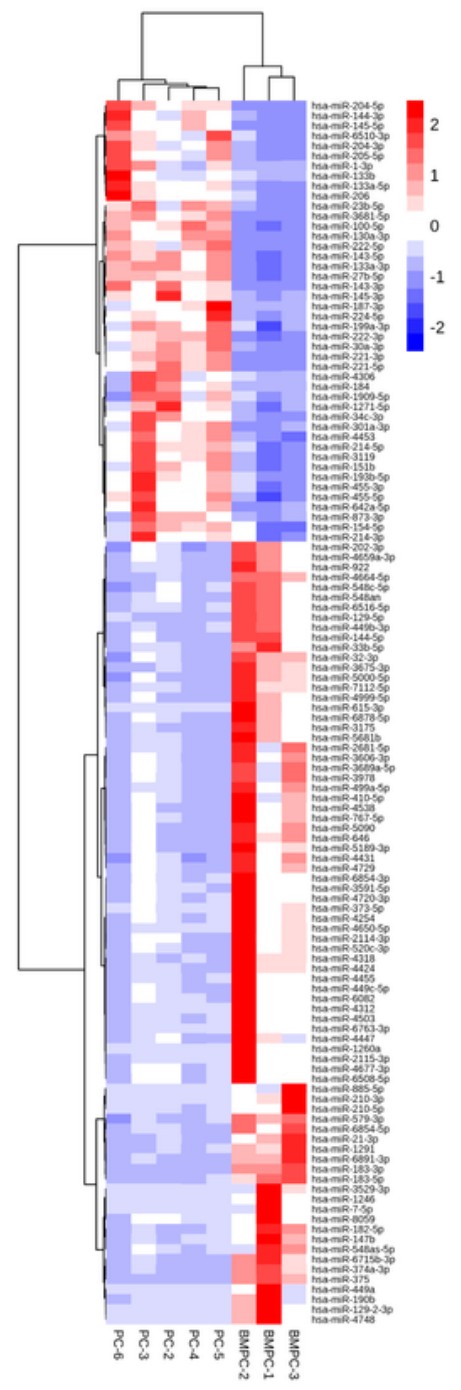

B

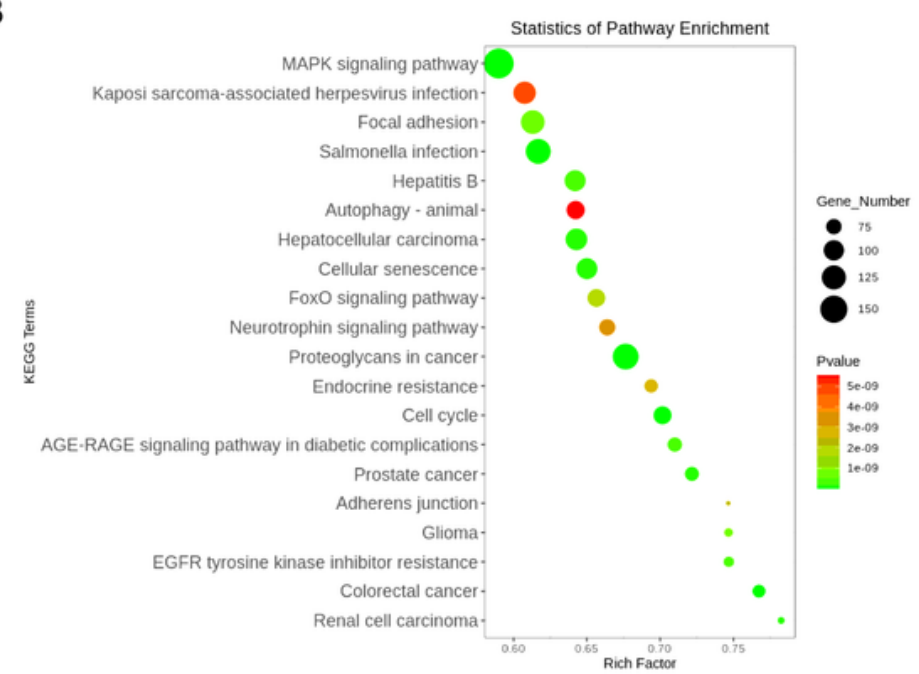

C

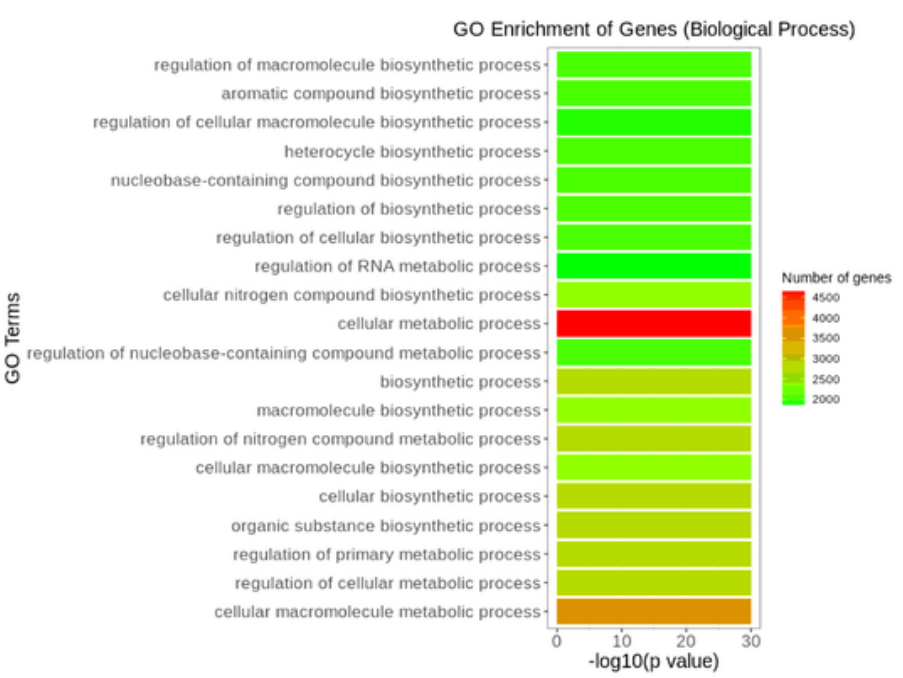

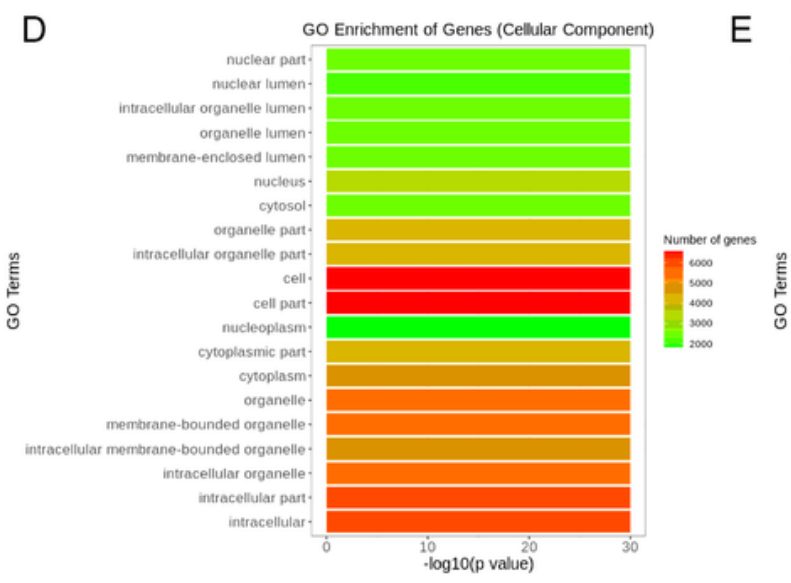

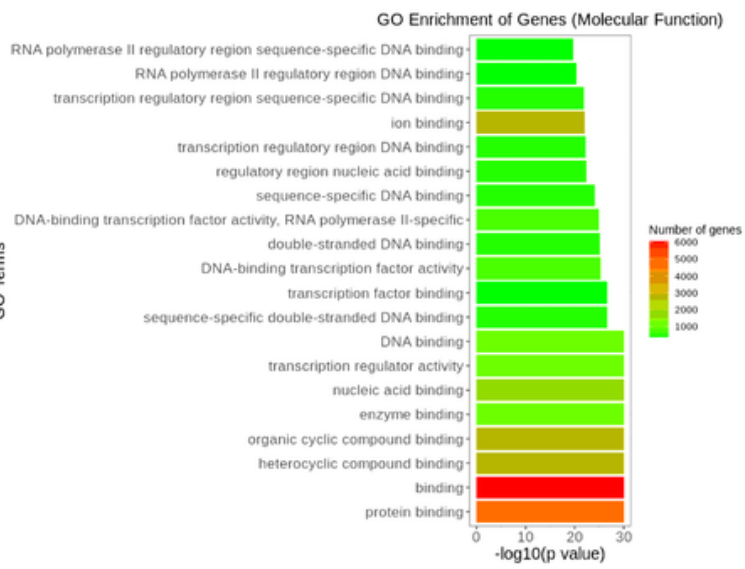

Figure 2

Gene expression profile in prostate cancer bone metastasis and prostate cancer without bone metastasis. (A) Heat maps showing the expression levels of miRNAs in each clinical tissue. The right column shows the genes. The red pixels show an increased level of the gene in each sample, whereas the blue pixels 
indicate decreased levels. (B) KEGG pathway in enrichment analysis of target gene of differently expressed miRNAs. $X$ axis represents the enriched factor and $Y$ axis represents the KEGG pathways. The color of the dot means the different P-value. The size of the dot indicates the gene number. (C-E) GO analysis of differently expressed miRNA target genes identified in prostate cancer bone metastasis. The digitals on the $y$-axis show the enrichment terms respectively. $X$ axis represents the $P$-value. The color of the dot indicates the gene number.

A

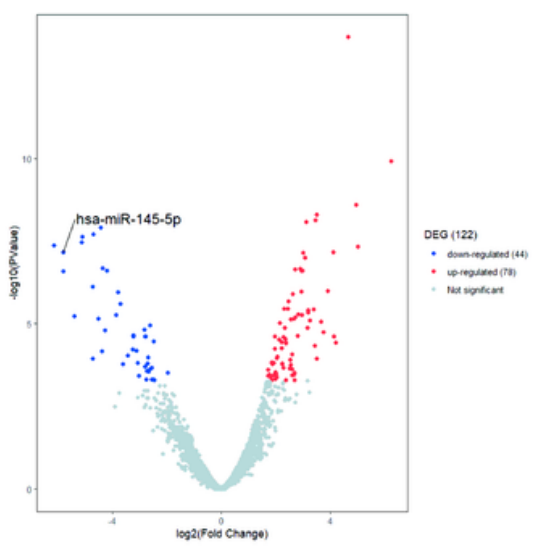

D

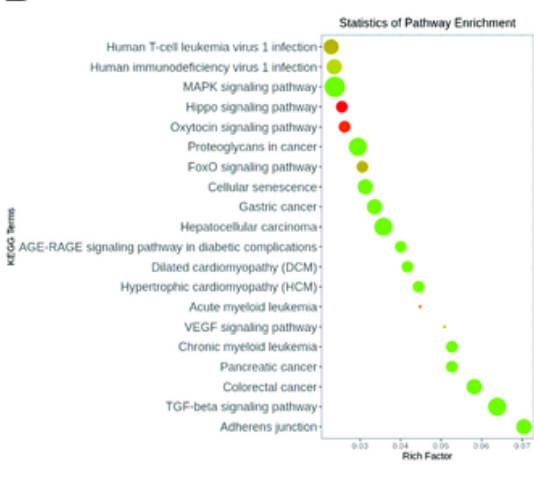

B

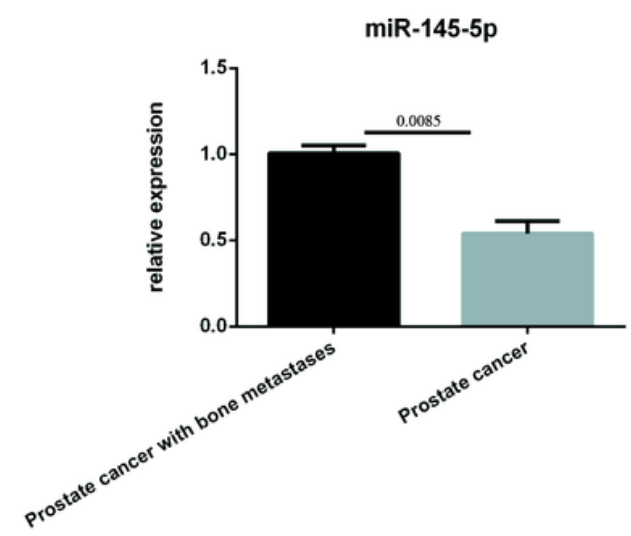

C

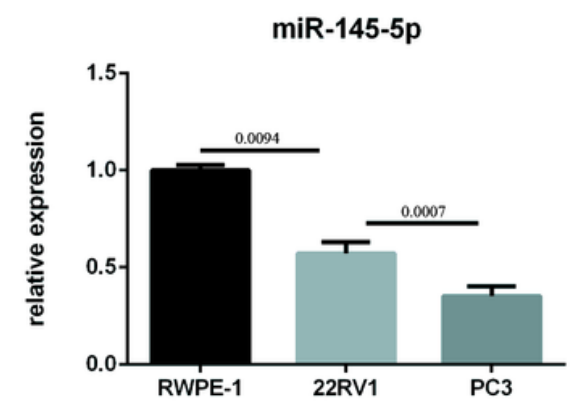

G

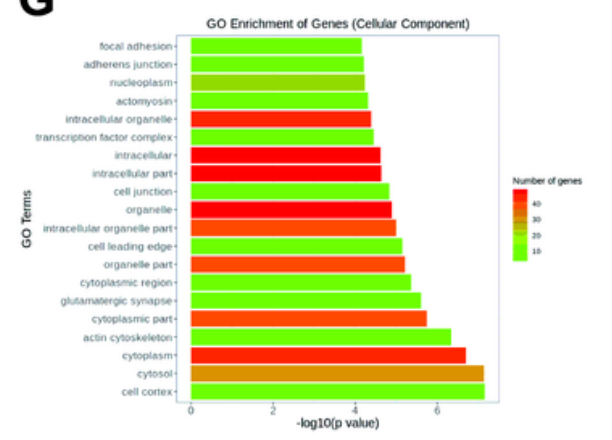

$\mathbf{E}$

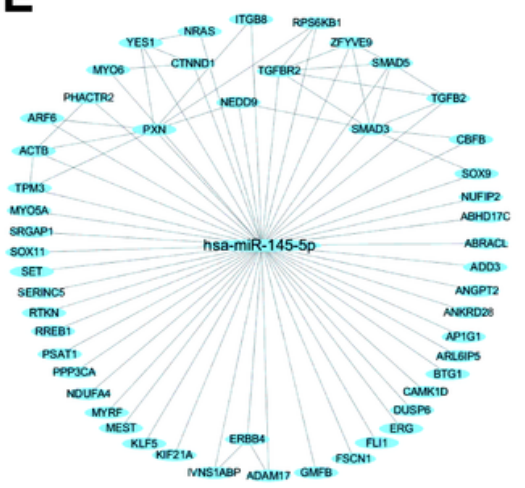

H

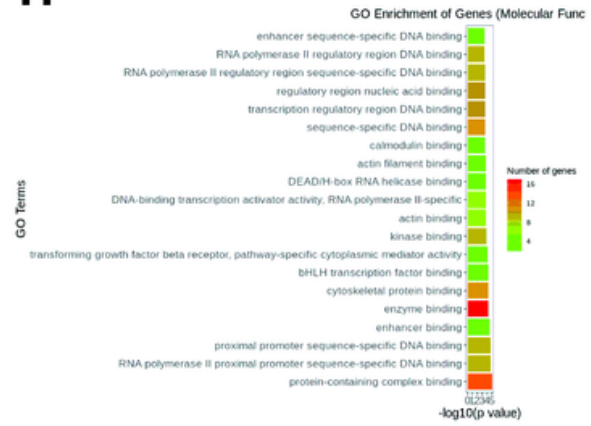

$\mathbf{F}$

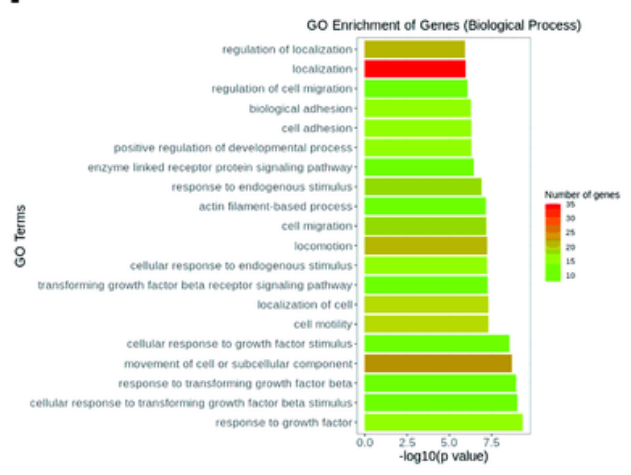

\section{Figure 3}

Functional annotation of miR-145-5p in prostate cancer bone metastasis. (A) The expression level of miR$145-5 p$ is decreased in prostate cancer bone metastasis (at least 2.0-fold changes and FDR<0.01) detected by next-generation sequencing. (B) The expressions of miR-145-5p in prostate cancer bone 
metastasis tissues detected by RT-qPCR. ( $n=10$, mean \pm SEM, two-tailed unpaired Student's t-test). The expressions of miR-145-5p in bone metastatic prostate cancer cell line (PC3), prostate cancer without metastatic cells (22RV1) and normal prostate epithelial cells (RWPE-1). detected by RT-qPCR. ( $n=3$ biological replicates, mean \pm SEM, two-tailed unpaired Student's t-test). (D) KEGG pathway in enrichment analysis of target gene of miR-145-5p. $X$ axis represents the enriched factor and $Y$ axis represents the KEGG pathways. The color of the dot means the different $P$-value. The size of the dot indicates the gene number. (E) miR-145-5p -mRNA negative correlation network. (F-H) GO analysis of miR-145-5p target genes identified in prostate cancer bone metastasis. The digitals on the $Y$-axis show the enrichment terms respectively. $\mathrm{X}$ axis represents the $\mathrm{P}$-value. The color of the dot indicates the gene number.

A

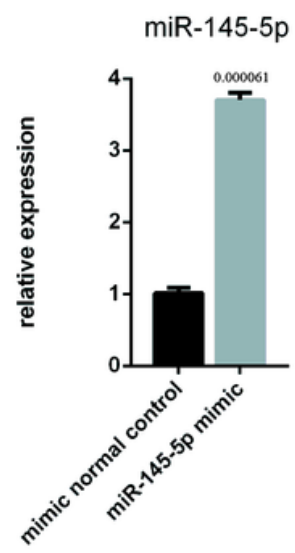

B

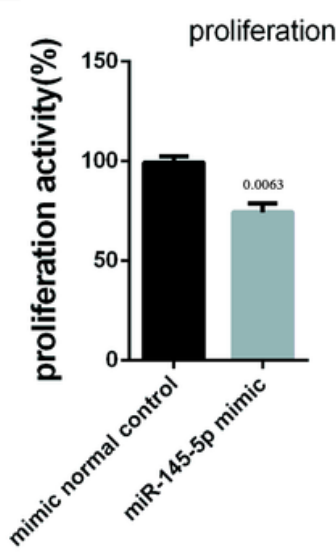

C mimic normal control

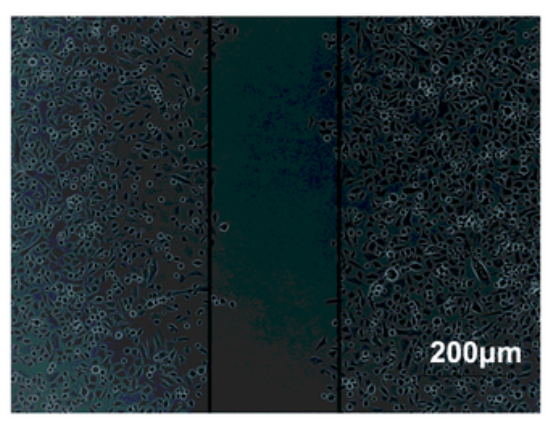

E mimic normal control
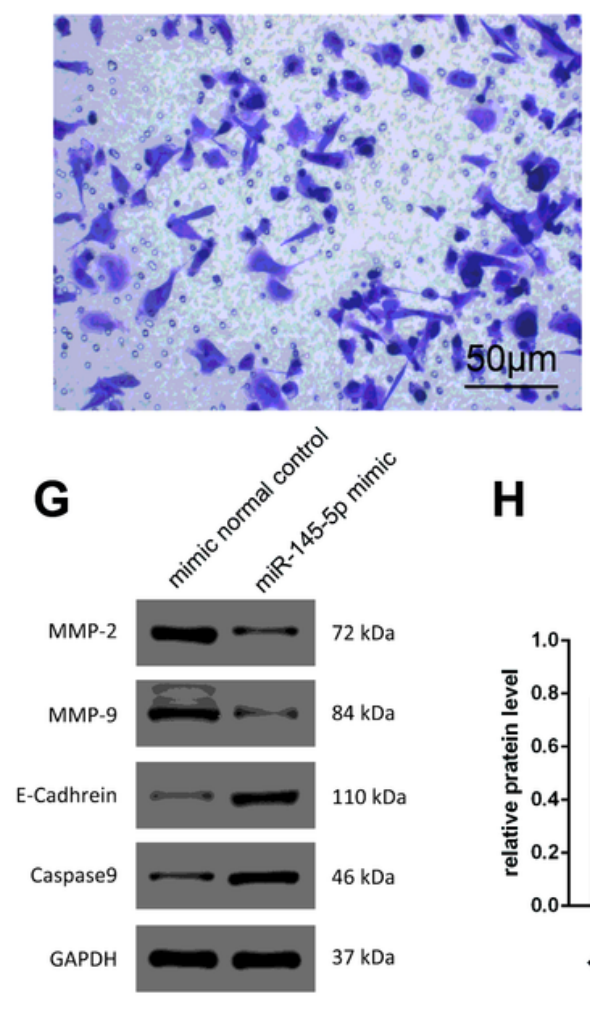

H
miR-145-5p mimic

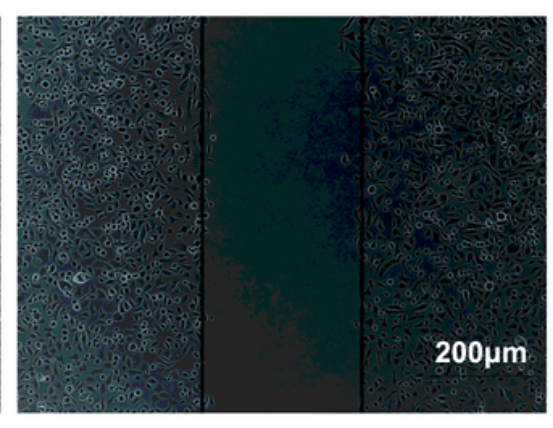

miR-145-5p mimic
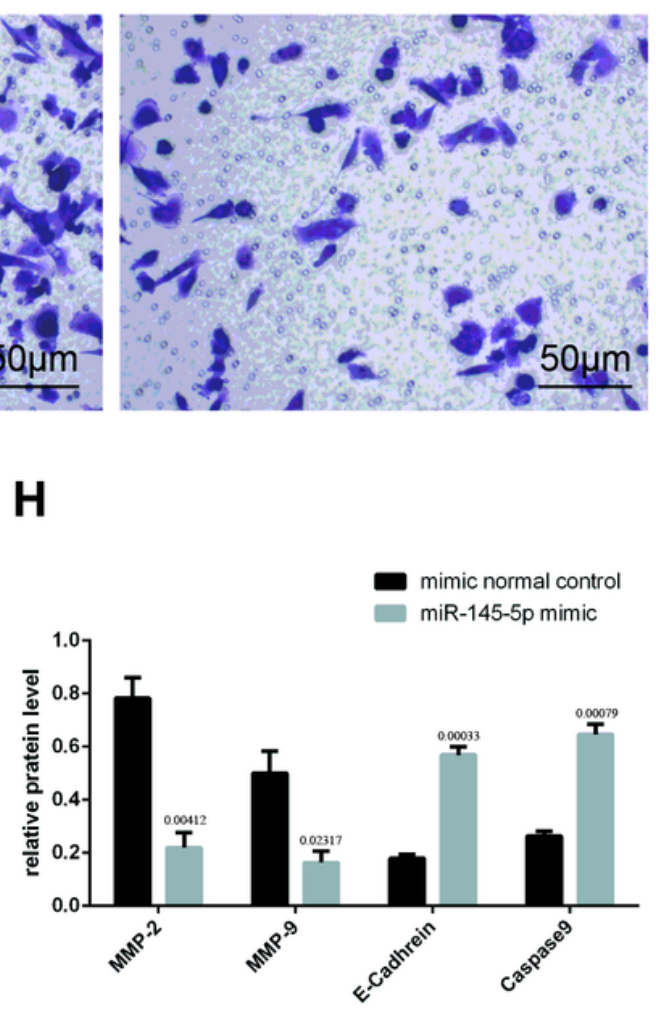

Figure 4 
miR-145-5p inhibits the proliferation, migration and invasion of in bone metastatic prostate cancer cell via epithelial mesenchymal transition. (A) The expression level of miR-145-5p after transfection mimics in PC3 cells. ( $n=3$ biological replicates, mean \pm SEM, two-tailed unpaired Student's t-test). (B) The proliferation rate of PC3 after transfection mimics. $(n=3$ biological replicates, mean $\pm S E M$, two-tailed unpaired Student's t-test). (C-D) The migration of PC3 cells after transfection mimics detected by scratch analysis. ( $n=3$ biological replicates, mean \pm SEM, two-tailed unpaired Student's t-test). ( $E-F)$ The invasion of PC3 cells after transfection mimics detected by transwell assays. $(n=3$ biological replicates, mean \pm SEM, two-tailed unpaired Student's t-test). (G-H) The protein levels of MMP-2, MMP-9, E-cadherin and caspase 9 in PC 3 cells after transfection mimics. ( $n=3$ biological replicates, mean \pm SEM, two-tailed unpaired Student's t-test). 


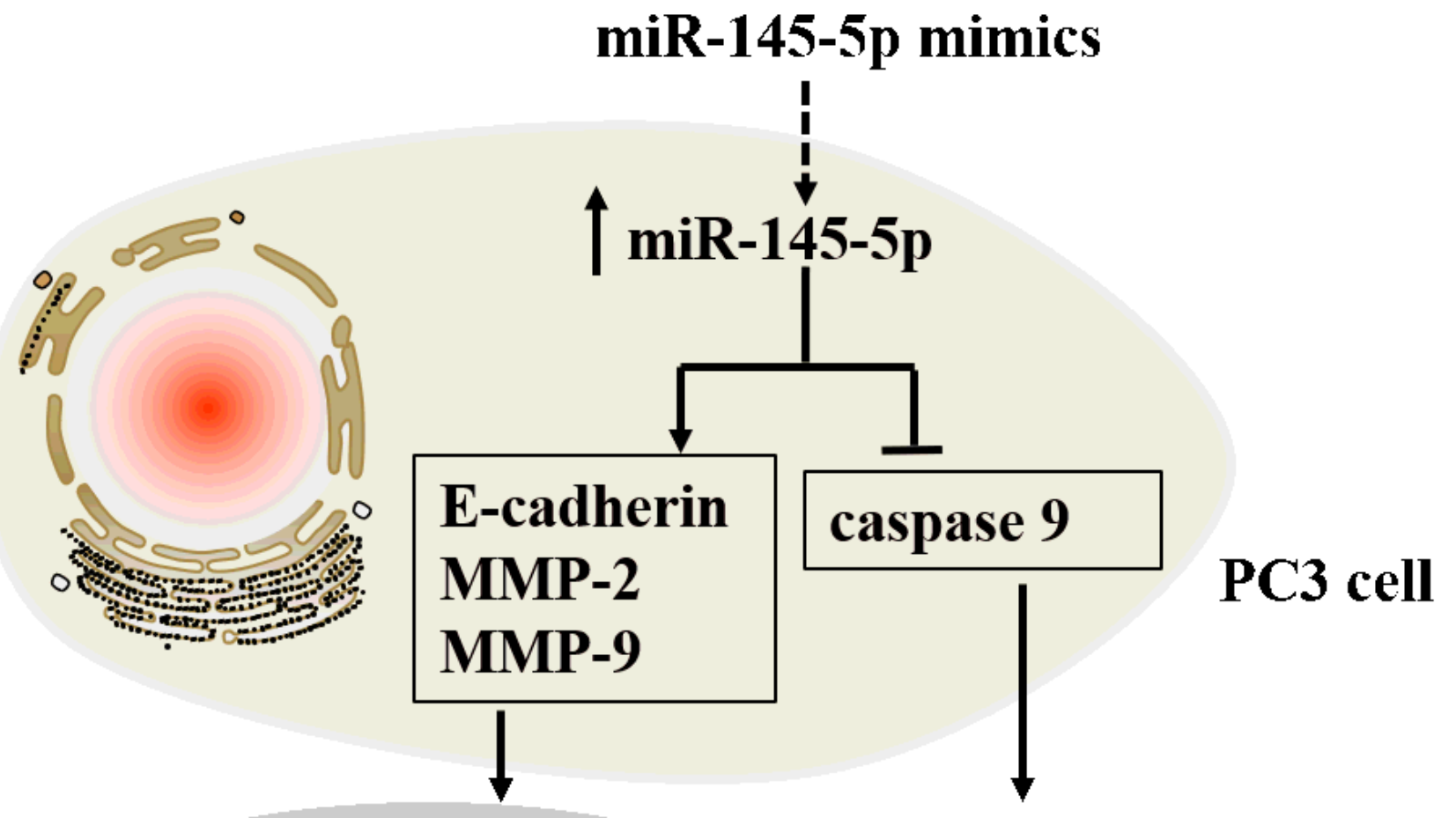

\section{epithelial mesenchymal transition}

\section{apoptosis}
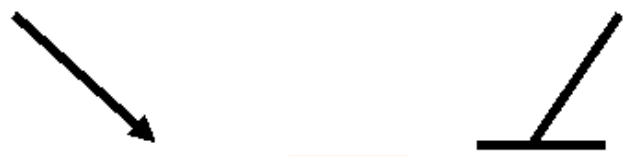

proliferation, migration

and invasion

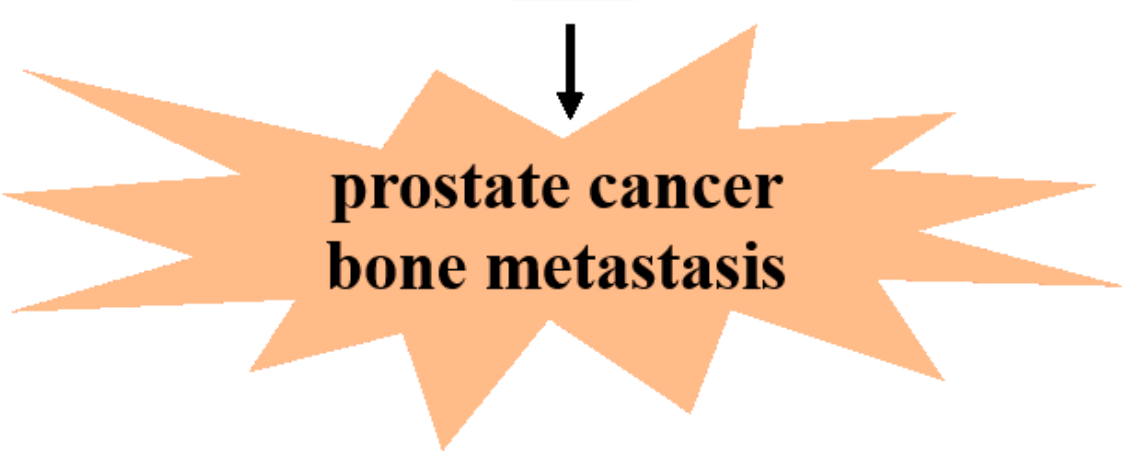

Figure 5

miR-145-5p mimic inhibits the progress of prostate cancer bone metastasis and potentially exerts a regulatory effect on prostate cancer bone metastasis. 